\title{
Xenopus: An Undervalued Model Organism to Study and Model Human Genetic Disease
}

\author{
Martin Blum Tim Ott \\ Institute of Zoology, University of Hohenheim, Stuttgart, Germany
}

\section{Keywords \\ Xenopus - Disease modeling · Analysis of human disease alleles - Model organisms of human disease - Ciliopathy . Congenital heart disease $\cdot$ Cilia $\cdot$ Left-right asymmetry}

\begin{abstract}
The function of normal and defective candidate genes for human genetic diseases, which are rapidly being identified in large numbers by human geneticists and the biomedical community at large, will be best studied in relevant and predictive model organisms that allow high-speed verification, analysis of underlying developmental, cellular and molecular mechanisms, and establishment of disease models to test therapeutic options. We describe and discuss the pros and cons of the frog Xenopus, which has been extensively used to uncover developmental mechanisms in the past, but which is being underutilized as a biomedical model. We argue that Xenopus complements the more commonly used mouse and zebrafish as a time- and cost-efficient animal model to study human disease alleles and mechanisms.

(c) 2018 S. Karger AG, Basel
\end{abstract}

\section{Introduction}

Early researchers studying amphibian embryology collected specimens from the wild. They depended on environmental parameters they could not control, such as the weather in the spring when amphibians lay their eggs in ponds and puddles [Hamburger, 1988; Gurdon and Hopwood, 2000]. The frog Xenopus made its first appearance as an embryological laboratory animal in the early 1900 s in the UK, when it first caught the attention of zoologists who found they could breed specimens in aquariums. Its call to fame happened in the 1940s and 1950s, when pregnancy testing was performed by injecting the urine of potentially pregnant women under the skin of female frogs: if gonadotropin was present, females reacted with egg-laying [Gurdon and Hopwood, 2000]. Xeno-

\section{Abbreviations used in this paper}

CHD congenital heart disease

LRO left-right organizer

MCCs multiciliated cells

MO morpholino oligomers

PCD primary ciliary dyskinesia

\section{KARGER}

(C) 2018 S. Karger AG, Basel

E-Mail karger@karger.com

www.karger.com/cto
Martin Blum

Institute of Zoology, University of Hohenheim

Garbenstrasse 30

DE-70599 Stuttgart (Germany)

E-Mail martin.blum@uni-hohenheim.de 


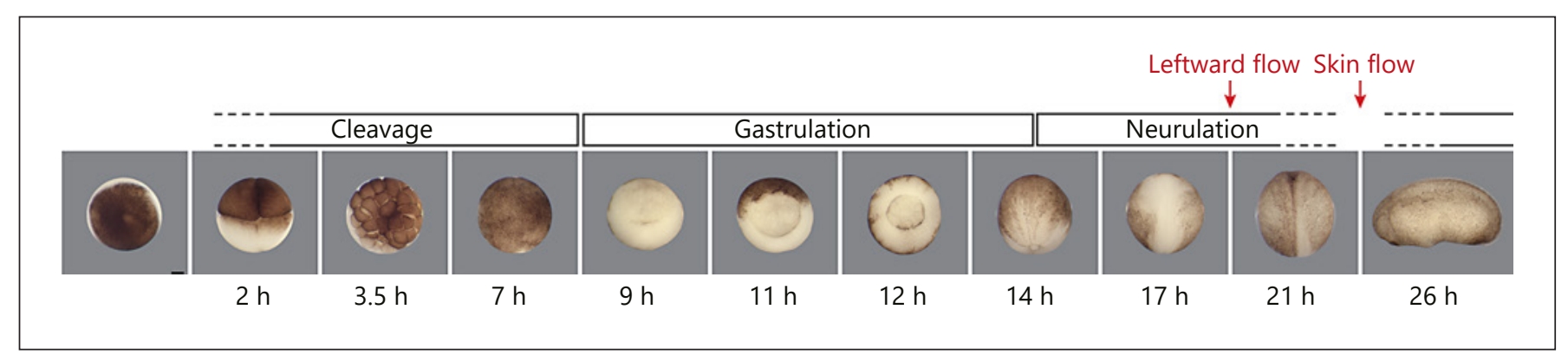

Fig. 1. Development of $X$. laevis embryos from the fertilized egg to the 5-day-old tadpole. Developmental stages, times, and key time points for the differentiation of left-right asymmetry, kidney, brain ventricle, and skin cilia are indicated. Scale bar, $250 \mu \mathrm{m}$.

(Figure continued on next page.)

pus frogs in developmental biology research were introduced in the early 1950s; the most famous and celebrated experiment from this period no doubt was John Gurdon's demonstration of the pluripotency of the somatic nucleus [Gurdon et al., 1958]. Gurdon cloned the very first vertebrate by nuclear transfer into a denucleated fertilized zygote, an experiment acknowledged by a Nobel prize some 54 years later in 2012. In the last 3 decades of the 20th century, Xenopus was widely used in laboratories worldwide, and a great many fundamental cell biological and developmental concepts were derived from work with frog embryos, most importantly the molecular regulation of the cell cycle [reviewed in Philpott and Yew, 2008], the first identification of numerous key vertebrate developmental genes, including those expressed in Spemann's organizer [reviewed in De Robertis et al., 2000], and the fundamentals of many signaling pathways, most prominently Wnt and BMP signaling [reviewed in Cruciat and Niehrs, 2013; Bier and De Robertis, 2015]. As such, the value and versatility of Xenopus as a model organism for vertebrate developmental mechanisms has been proven and never debated.

However, when it comes to evaluating human disease alleles, most clinicians and human geneticists turn to the mouse and to zebrafish, which are considered the bona fide model organisms to study human disease mechanisms. Both were established from the start as genetic models, whereas Xenopus - and amphibians in general excel as experimental models. However, with the development of the diploid $X$. tropicalis as a laboratory animal, the complete sequencing of the $X$. laevis and $X$. tropicalis genomes [Hellsten et al., 2010; Session et al., 2016], and the advent of genome editing, Xenopus has also become a member of the family of genetic model organisms. A number of transgenic lines are already available from the
US and European resource centers (Xenbase.org; xenopusresource.org), and new ones can be generated fairly quickly [Aslan et al., 2017; Moreno-Mateos et al., 2017]. Candidate genes and alleles for human genetic disease are being identified at an enormous pace: analyses such as genome-wide association studies, GWAS, whole-exome sequencing, WES, and DNA and RNA sequencing from healthy and diseased tissues generate long lists of candidate genes and alleles that overwhelm biomedical researchers with the task of evaluating large data sets rapidly and at a manageable cost [MacArthur et al., 2014; Samocha et al., 2014; Getwan and Lienkamp, 2017]. The demand is for models to verify or discard candidate genes and to establish predictive models in which therapeutic options can be developed and tested.

Every model system, of course, has its benefits and its limitations. The mouse, for example, as a mammal has a very similar genome to that of humans and has an enormous tool box available for genetic manipulations. However, mouse models require costly investments in animal housing and maintenance, litter sizes are small with on average 8 offspring, only a fraction of which will carry the mutant allele, and the analysis of mutant mice is time consuming. Zebrafish produce large numbers of offspring, are less expensive to house, and have well-developed genetic approaches and many available mutant and reporter lines. However, the teleost genome duplication has led to the sub-functionalization of many biomedically relevant genes and a loss of synteny compared to mammals [Garcia de la Serrana et al., 2014]. The possibility to directly observe embryonic development and even perform live cell imaging has been extensively used with great success in zebrafish. However, this is not a unique feature of zebrafish; kidney development and neural crest cell migration, for example, have been directly imaged in live frog em- 


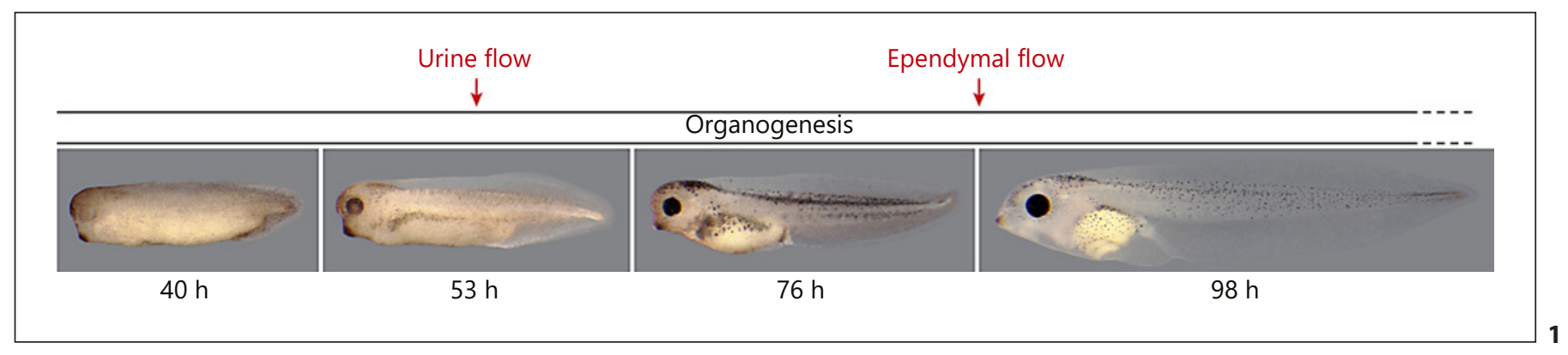

bryos at great temporal and spatial resolution [Lienkamp et al., 2010, 2012; Szabó et al., 2016; Getwan and Lienkamp, 2017]. Zebrafish also have certain organ-specific limitations. For example, they cannot model human syndromes that affect the limbs or digits, the lungs or the diaphragm, structures that are involved in many human congenital syndromes but are absent from fish. In addition, although the zebrafish heart has been an excellent model for studying regeneration, having just one atrium and one ventricle cannot fully model malformations of the human four-chambered heart. The frog Xenopus, as described below, has great potential to complement the more established mouse and zebrafish, and thus to greatly enhance the repertoire of predictive animal models of human disease. This review describes the many experimental attributes of Xenopus that enable detailed studies of candidate genes, with the intention of alerting clinical scientists and human geneticists of the potential of this established and still relevant, seminal animal model.

\section{The African Frog, Xenopus}

Two frog species are used in research these days, $X$. laevis (South African clawed frog), which has been utilized for decades, and X. tropicalis (Western clawed frog), which has only been introduced recently in many laboratories [Tandon et al., 2017]. X. tropicalis is a genetically diploid species that is raised at temperatures of $24-26^{\circ} \mathrm{C}$, whereas X. laevis, which prefers colder waters of $18^{\circ} \mathrm{C}$, is the product of a fertile hybridization of two species, i.e., contains a duplicated set of genes and chromosomes. Frogs as amphibians, of course, are distant from humans genetically, but they share a common tetrapod ancestry that is closer in evolutionary time than bony fish. The complete genome sequencing and careful annotation of both species reveal an astonishingly high degree of synteny with humans; even in the duplicated genome of $X$. laevis, because the two sets of chromosomes do not crossover, there has been less sub-functionalization of duplicated copies [Hellsten et al., 2010; Session et al., 2016; Harland and Gilchrist, 2017]. Close to $90 \%$ of human disease genes have homologs in both Xenopus species, and sequence conservation is generally high, a prerequisite for any predictive animal model. Many laboratories use both species in parallel, i.e., use X. tropicalis for genome editing and $X$. laevis for most other studies.

A central feature of this model, which has rendered it attractive in the past and shows great promise for future use, is the ease of gaining access to eggs and embryos: females lay large clutches of thousands of eggs and egg laying is inducible by hormone priming; researchers nowadays capitalize on the same principle that made the frog a system for pregnancy testing. Eggs can be fertilized by natural mating or artificially by the addition of sperm from testes, which in the case of $X$. laevis can be kept for days in the refrigerator without losing fertilization capacity. Eggs in addition are large, with diameters of 1.2 and $0.8 \mathrm{~mm}$ in $X$. laevis and $X$. tropicalis, respectively. This allows easy intracellular microinjections, either into the zygote or into defined blastomeres of early developmental stages, up to about the 64-cell stage, when cells become too small to be readily targeted. Furthermore, the lineage of individual cells is well known [Jacobson and Moody, 1984; Moody, 1987, 2000], i.e., injections can be very precisely targeted to tissues and organs at later developmental stages, up to the late tadpole stage, just before metamorphosis, which is reached after 5 days of development and in which organogenesis is basically completed (Fig. 1). Thus, precise manipulations can be performed during early cleavage stages in the first few hours of development, and consequences thereof be followed continuously for the next several days, throughout organ development. It is therefore possible to manipulate large numbers of embryos that develop very rapidly to the desired stages of organogenesis, making it possible to obtain 

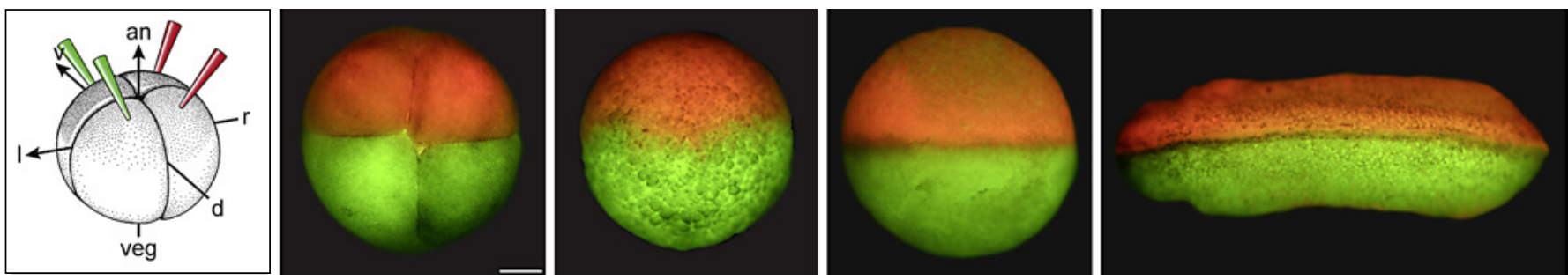

Fig. 2. Precise targeting of injections to the left and right side of the developing Xenopus embryo. A green and a red fluorescent dye were injected into left and right blastomeres at the 4-cell stage, respectively (scheme on left and photograph in second panel). This separation persists throughout development, as shown in a blas-

results within a short time frame. Because the females will lay high quality eggs for many rounds per year, and housing is relatively inexpensive, animal costs are much lower particularly compared to mouse.

A unique feature that is not found in any other animal model used for the assessment of human genetic disease these days is the possibility of one-sided injections, meaning that only one side of the embryo is manipulated with the contralateral side serving as an internal control (Fig. 2). This attribute, which is a consequence of the first cleavage division separating the left and the right side of the embryo, is quite important when assessing the phenotypic consequences of experimental manipulations, because the variation in wild-type gene expression can sometimes be sizable across different clutches of embryos. The ability to manipulate a very large number of embryos per experiment and the possibility of selecting specimens for analysis based on the normal development on the control side adds a high level of rigor, statistical significance, and reproducibility to every study.

The large size of eggs and embryos, which distinguishes Xenopus frogs from both zebrafish and mouse, at least during the first third of embryonic development, offers one more unique advantage: enough material to perform proteomic analyses with a manageable number of specimens [Nagasawa et al., 2013; Amin et al., 2014; Wühr et al., 2014, 2015; Peshkin et al., 2015]. With the rapid advance of proteomics, this characteristic will certainly become more prominent in the near future and for example allow comparative interaction studies of wild-type and mutated proteins. A last feature deserves mentioning, as it comes unexpected to many who do not know the frog: there are stem cells and they can be easily differentiated to develop into defined tissues and organs (Fig. 3). These naive cells can be readily excised from the embryo at the end of cleavage divisions, before gastrulation and the tula stage embryo at the end of cleavage divisions, a neurula embryo, and a tadpole. This allows one-sided manipulations, i.e., the non-manipulated side can be used as an internal control. Scale bar, $250 \mu \mathrm{m}$.

specification of the germ layers begin. They reside at the animal pole, i.e., the pole pointing away from the gravity vector. Isolated so-called animal caps differentiate into undefined atypical ectoderm-like cells upon culture in a dish. However, the addition or injection of defined growth factors, transcription factors, or other inducers such as retinoic acid transforms these cells into all kinds of tissues, be it kidney, heart, or pancreas (Fig. 3) [reviewed in Asashima et al., 2009; Kurisaki et al., 2010; Hosoya et al., 2012]). In addition, morphogenetic movements can be studied, as explants may undergo what is called convergence extension [Keller et al., 2000; Keller, 2002], i.e., elongation due to the intercalation of cells (Fig. 3), a pronaling, with relevance to many human diseases [reviewed in Xiao et al., 2017].

Intracellular microinjections are the single most important technique when using the frog model. The setup is fairly simple: a dissecting microscope fitted with a flexible light source and a device to hold and direct the injection needle. Hundreds of embryos can be injected on any given day. Most labs have a number of injection setups to allow the optimal use of egg clutches, which develop in synchrony and thus need to be injected during limited time windows of $30-45 \mathrm{~min}$ or so. Manipulations in many instances concern gene expression; antisense morpholino oligomers (MO), i.e., single-stranded nucleotide chains harboring a morpholino ring instead of ribose or deoxyribose, bind to mRNAs and interfere with translation, if they bind at the start AUG (translation-blocking MOs, or TBMOs), or with splicing, when they cover a splice donor or acceptor site (splice blocking, or SBMO) [Heasman, 2002]. The correct targeting to the tissue or organ of interest is usually monitored by co-injecting lineage tracers, for example fluorescent dextran or membrane-targeted GFP, that allow one to discard mistargeted specimens by cess that is under the control of non-canonical Wnt sig- 


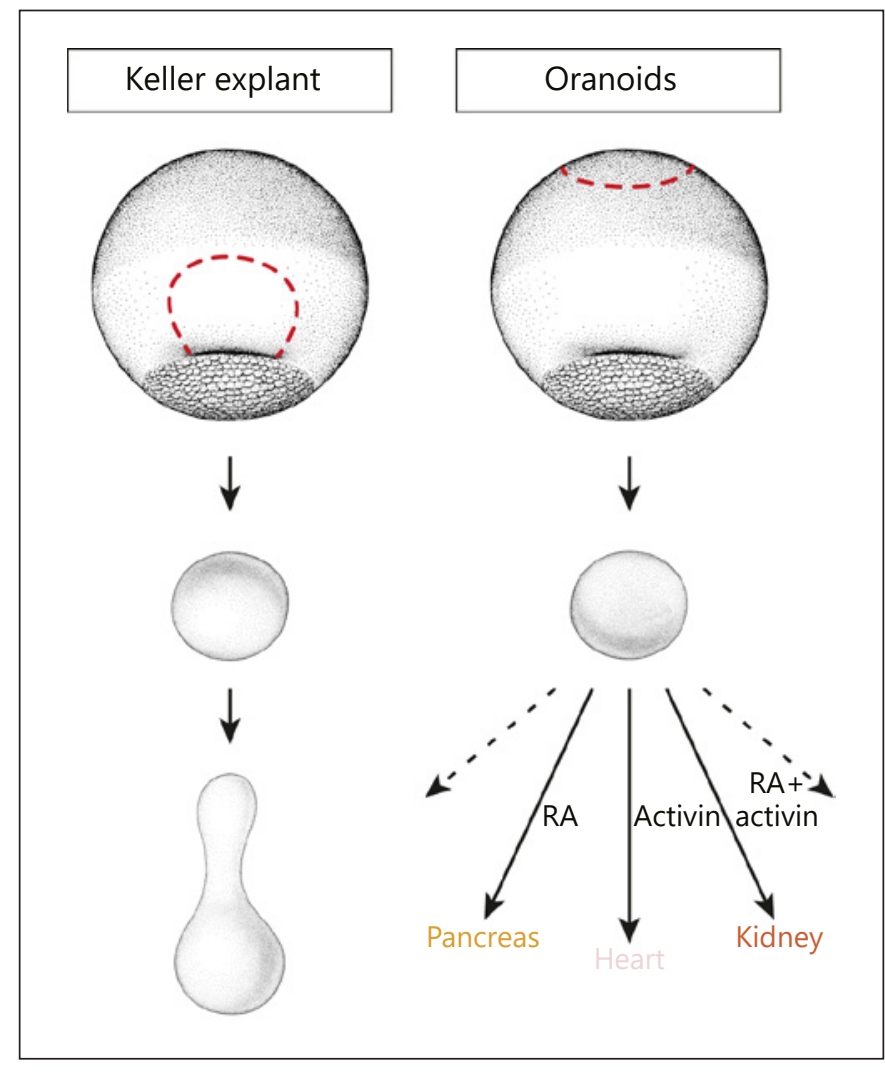

Fig. 3. Explant culture systems to study morphogenetic movements (convergent extension). Left: so-called Keller explants, which contain the Spemann organizer region and therefore elongate (extend and converge) in culture. In this system, non-canonical Wnt signaling can be studied and manipulated by injecting for example mRNAs into this region at the 4-cell stage and explanting at the beginning of gastrulation. Right: animal cap explants can be differentiated by injection or culture with inducers to become pancreas, heart, and kidney tissues, among other options available to the investigator [see Asashima et al., 2009].

just looking down the microscope. The specificity of MOs of course needs to be carefully controlled, as off-target effects cannot be excluded a priori, as with all manipulations that require interactions with specific nucleotide sequences, be it genomic or at the RNA level. Controls include the use of more than one $\mathrm{MO}$, dose dependency, and rescue of phenotypes by co-injecting rescue mRNAs that are not targeted by the MO. The debate on specificities of gene and mRNA targeting is old and ongoing and should remind every researcher to remain critical and careful when interpreting a specific result [Eisen and Smith, 2008; Blum et al., 2015; Kok et al., 2015; Moulton, 2017; Gentsch et al., 2018]. In Xenopus, genome editing by the CRISPR/Cas9 technology is easily performed and

Xenopus: An Undervalued Model

Organism for Human Genetic Disease can serve to validate the use of MOs, as well as being utilized as a valid genetic tool in its own rights [Garfinkel and Khokha, 2017; Naert et al., 2017; Tandon et al., 2017; Blitz, 2018; Naert and Vleminckx, 2018]. With respect to the analysis of candidate human disease genes and alleles, both techniques are successfully used (see below). The interaction of genes, however, which is of central importance when studying the molecular mechanisms underlying diseases, is studied with great ease by using MOs [Blum et al., 2015]: doses are selected for the two or three genes in question, which on their own do not result in any phenotypic alteration of development. Defects arising from their combined injections testify to genetic interactions, in much the same way as the analysis of compound heterozygotes in mouse and zebrafish (or invertebrate models such as Drosophila). Dosing of phenotypic effects in addition offers the advantage of circumventing early embryonic lethality and thus studying genes at developmental stages at which corresponding mutants are not available for analyses. Many human congenital syndromes are caused by a combination of heterozygous hypomorphic alleles, and thus better studied by reducing protein levels, compared to genetic null alleles. In addition, mutant human alleles can be easily expressed in Xenopus embryos, simply by microinjection of mutant mRNAs. In this way, disease alleles can be functionally assessed, either in wild-type embryos or in specimens in which the endogenous gene was knocked down by antisense MOs [Blum et al., 2015].

\section{Suitability of Xenopus to Study Human Malformations and Disease}

Over the past decade or so, the international Xenopus community has witnessed a change in focus, from studying primarily basic developmental and cell biological mechanisms to analyzing processes related to disease mechanisms in the wider sense. This may reflect policies of funding agencies such as in the USA, where translational research is more likely to receive funding than pure basic science, or just because of the biomedical task and because the frog has that potential to add knowledge in a substantial manner. It is beyond the scope of this review to give a full account of activities and successes. Examples of cases in which disease mechanisms have been elucidated by studies in Xenopus are detailed on the community website (http://www.xenbase.org/community/static/xenopuswhitepaper/2016/2016-XenopusWhitePaper-Final.pdf), which in addition provides a wealth of information for 


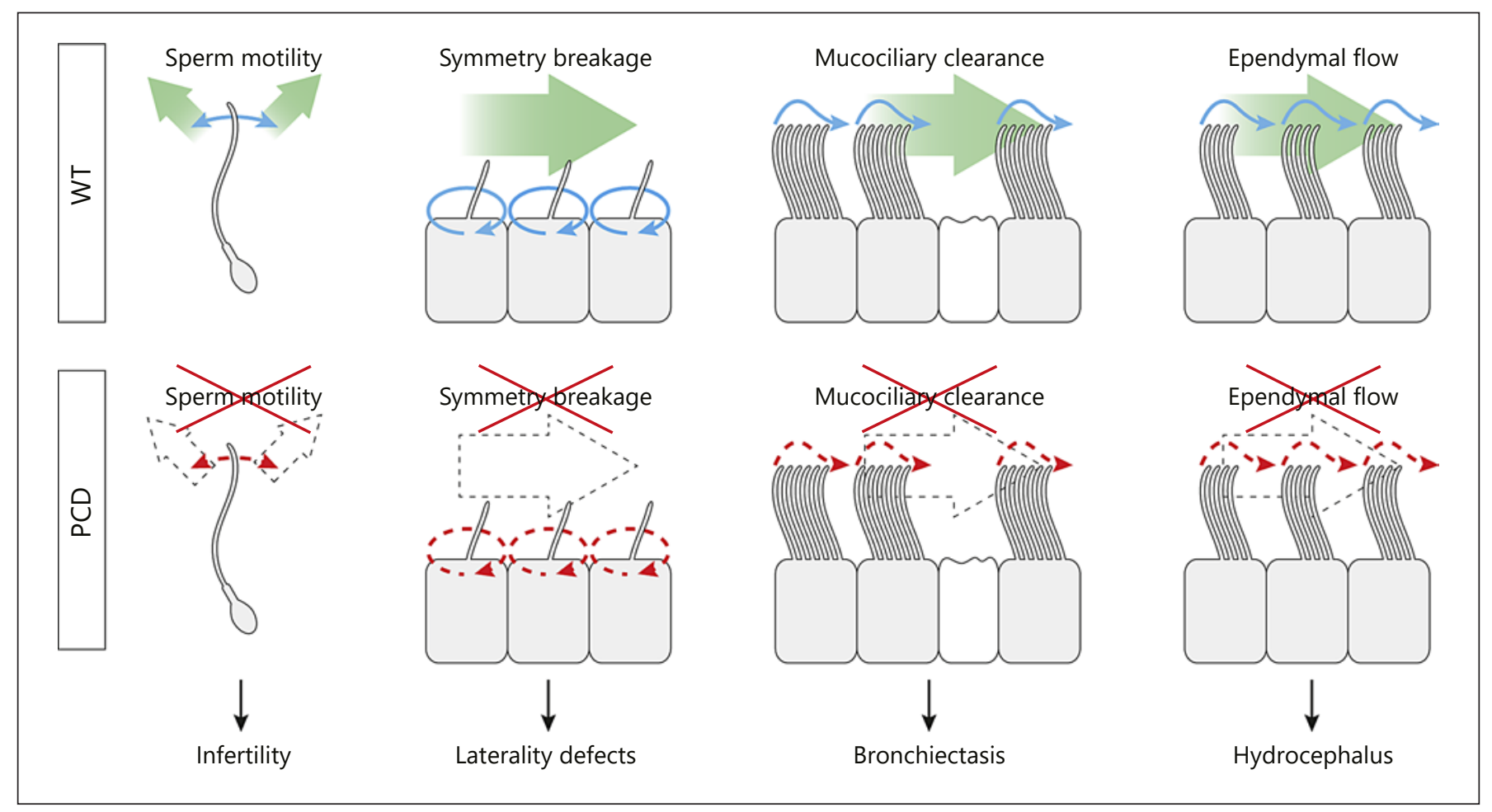

Fig. 4. Summary of PCD phenotypes. Except for sperm mobility, all others are easily studied in Xenopus embryos.

Xenopus researchers at large. It is worth citing a few examples, however, such as, among others, the work of Bruno Reversade at the Institute of Medical Biology in Singapore, who uses Xenopus alongside other model organisms, be it vertebrate or invertebrate, to unravel mechanisms of human diseases. Autosomal recessive cutis laxa, for example, a syndrome associated with - among others - lax and wrinkled skin, is caused by mutations in PYCR1, which encodes an enzyme involved in proline metabolism. Knockdown of the orthologous gene in Xenopus resulted in hypoplasia and wrinkles of the tadpole skin, i.e., was able to model the human disease [Reversade et al., 2009]. Mutations in the homeodomain transcription factor IRX5 impair craniofacial development and germ cell migration in humans. When the Xenopus ortholog was knocked down, migration of progenitor cells in the branchial arches and gonads was disrupted, faithfully reproducing the human disease [Bonnard et al., 2012]. A last and spectacular example from the Reversade laboratory concerns mutations in SMCHD1, which encodes an epigenetic regulator. Patients suffer from an extremely rare syndrome in which the nose is completely absent. Biochemical studies in Xenopus embryos demonstrated that disease mutations resulted in gain-of-function alleles, a finding that would not have been possible by just pursuing loss-of-function approaches [Gordon et al., 2017]. Pediatricians Martina Brueckner and Mustafa Khokha at Yale have systematically identified genes and alleles in children suffering from congenital heart disease (CHD) [Fakhro et al., 2011; Boskovski et al., 2013; Endicott et al., 2015; del Viso et al., 2016; Griffin et al., 2018]. A series of high-profile publications has demonstrated that analyses of CHD candidate genes in Xenopus not only verified disease genes and mutations, but resulted in rather unexpected and spectacular findings, such as the identification of a protease that cleaves the Notch receptor in the process of specifying motile versus immotile cilia required for symmetry breaking and asymmetric heart morphogenesis [Boskovski et al., 2013]. Kidney disease is another very promising area in which Xenopus offers great potential, because the tadpole differentiates one fully functional nephron on either side, ideally suited to studying the various steps of kidney development from tubulogenesis to segmentation and active filtration, again in a one-sided manner with its internal control [Lienkamp et al., 2012; Hoff et al., 2013; Lienkamp, 2016]. Both these examples have recently been covered by excellent reviews, to which the interested reader is referred [Garfinkel and Khokha, 2017; Getwan and Lienkamp, 2017]. 
A large group of diverse genetic diseases that is particularly well qualified to be studied in Xenopus is represented by the so-called ciliopathies [Fliegauf et al., 2007; Mitchison and Valente, 2017]. Cilia protruding from the cell surface may act as antennas and harbor receptors for all sorts of extracellular signals (primary cilia), or they may be motile to propel the cell itself (such as the sperm cell) or the extracellular fluids surrounding the ciliated tissue [Marshall and Nonaka, 2006]. A great many diseases are caused by defective cilia of one kind or the other [Gerdes et al., 2009; Brown and Witman, 2014], with an estimated prevalence of up to one human patient in every 300-500 healthy individuals. These figures are preliminary, as cilia-relationships to diseases keep being discovered. In the following, we will focus on motile cilia, as this is an area of our own research and expertise. Human disorders resulting from cilia motility defects are summarized as primary ciliary dyskinesia (PCD) [Knowles et al., 2016; Bustamante-Marin and Ostrowski, 2017]. They mostly concern mucus clearance in the airways, where multiciliated cells (MCCs) beat in a coordinated manner to transport mucus, to which inhaled particles and pathogens adhere, upwards towards the pharynx (Fig. 4). To date, about 40 disease genes have been identified that explain some two-thirds of PCD in patients. It is expected that the so-far unexplained cases are caused by an additional large number of genes. Such disease candidate genes need to be assessed and Xenopus is an ideal model in which to do so [Walentek and Quigley, 2017].

Although aquatic and short of airways, the larval skin works the same way and fulfills the same function as the human airway: the epithelium harbors MCCs and cells that produce mucus [Dubaissi and Papalopulu, 2011; Brooks and Wallingford, 2014, 2015; Dubaissi et al., 2014; Walentek et al., 2014]. The skin serves as a first line of defense, i.e., pathogens adhering to the mucus are transported by coordinated ciliary movements that transport mucus along the specimen, from head to tail and off the tadpole (Fig. 4). Defective cilia render larvae susceptible to bacterial infections, a condition that is lethal during development [Dubaissi et al., 2014]. Skin MCCs can be easily visualized, histologically by staining the ciliary axonemes, and functionally by high-speed videography (Fig. 5; online suppl. movie 1; for all online suppl. material, see ww.karger.com/doi/10.1159/000490898). Motility can be easily manipulated as well, for example by knocking down dnah9, an axonemal dynein which is critically required for many motile cilia [Vick et al., 2009] (online suppl. movie 2). Ciliary defects such as those resulting from dnah 9 knockdown become immediately ap-

Xenopus: An Undervalued Model

Organism for Human Genetic Disease parent in larvae, before muscles are fully functional. Larvae, laying on one side, move in a hovering motion across the agar-coated dish in which they are cultured due to the ciliary beating of their skin MCCs. By measuring the speed of the hovering movement on the control side and then flipping over the individual and doing the same measurement on the experimental side, one can detect cilia defects without elaborate and expensive equipment [Vick et al., 2009]. By simply observing the hovering behavior of the embryo, one can screen for which manipulations should be followed by more time-consuming and expensive additional experiments, such as histological assessment of ciliary parameters (number, length, orientation, and motility) or high-speed time-lapse videography to analyze the beat pattern directly. Motility should be rescued upon coinjection of a wild-type mRNA not targeted by the MO. Any allele of a gene that affects ciliary motility upon loss-of-function can be tested in the same setting, i.e., by co-injecting the allele in question along with a blocking MO. Xenopus is the only vertebrate model which allows fast and non-invasive in vivo assessment of human PCD disease alleles, avoiding artifacts that can arise from cultured cells such as air-liquid interphase cultures. A graded response may become visible by these types of analyses as well, such as for example alleles that do not result in complete paralysis of ciliary motion but in altered beat patterns or slower motilities.

PCD patients, depending on the genes mutated and the nature of the mutations, may suffer from immotile cilia at other sites and organs as well. For some of these, such as sperm immobility, which is frequently encountered in humans [Inaba and Mizuno, 2016], using the Xenopus microinjection approach is not recommended because the manipulation does not carry over to the next generation without using a transgenic approach (which are possible but would take months and extensive permits). However, defects that are readily studied with the fast and efficient Xenopus approaches include laterality defects and hydrocephalus [Blum et al., 2009; Hagenlocher et al., 2013; Blum and Ott, 2018b]. Both are prominent in PCD patients and are caused by cilia defects at the left-right organizer (LRO) and in the embryonic brain ventricles, respectively [Zariwala et al., 2007].

The LRO is the first tissue in the developing embryo in which motile cilia are required [Blum et al., 2014b; Shinohara and Hamada, 2017]. A monociliated epithelium forms during gastrulation and is positioned in the dorsal midline of the neurula embryo, in the posterior notochord ("node") of mice, in Kupffer's vesicle in zebrafish, and at the roof of the primitive gut or archenteron in frogs 

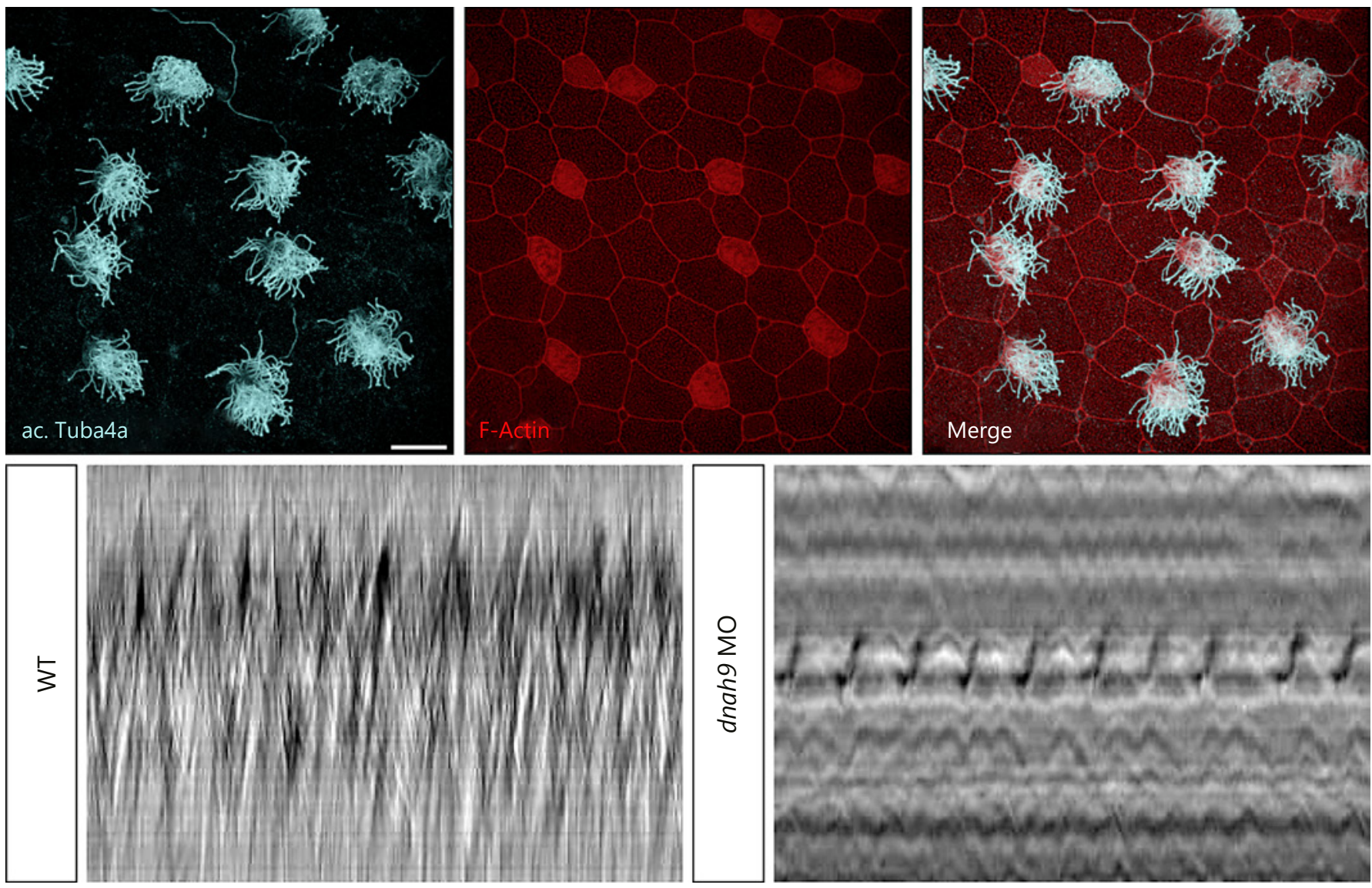

Fig. 5. The mucociliary epithelium of the larval skin. Top: immunofluorescence analysis of the larval epidermis, co-stained for cell boundaries (actin) with phalloidin and for cilia with an antibody directed against acetylated $\alpha$-tubulin. Bottom: ciliary motility, as

displayed by a horizontal kymograph of an epidermal ciliary tuft from a wild-type (left) or dnah 9 morphant embryo (right) to visualize ciliary beating over a 0.5 -s time period. Scale bar, $25 \mu \mathrm{m}$.

[Blum et al., 2007]. Although LROs differ somewhat in their morphology, they are homologous structures and involve homologous genes for their function [Blum et al., 2014a]. In Xenopus, the sequence of events leading to asymmetric organ morphogenesis and placement in the tadpole are particularly well understood and accessible to manipulation and analysis at all stages, from the foxj1dependent specification of the LRO precursor tissue to the leftward fluid flow at the LRO and to the left-asymmetric induction of the Nodal signaling cascade in the left lateral plate mesoderm, from which organ asymmetries ultimately result [Blum et al., 2014b]. Mutations in human PCD genes may or may not result in left-right defects; if they occur, they are readily detected along with other defects in the course of the same experiment: knockdown of the respective gene. This analysis will reveal another defect if present, namely hydrocephalus, i.e., ex-

panded brain ventricles which - among other causes may arise from defective brain MCCs (Fig. 4). Brain flow produced by these MCCs can be imaged in Xenopus tadpoles [Hagenlocher et al., 2013], and ciliary parameters, such as length and polarization, are equally accessible. Thus, except for sperm motility, the entire range of PCD defects is accessible for analysis in Xenopus tadpoles in a matter of days, once the tools for manipulation are available and have been tested for specificity.

Cilia function related to PCD in most cases (airways, LRO, brain) require oriented arrangement of cilia in order to produce a directed flow of extracellular fluids. Cilia orientation is governed by the planar cell polarity pathway, under the control of non-canonical Wnt signaling [Shinohara and Hamada, 2017]. The orientation of the PCP vector, however, is under biomechanical control, namely physical strain exerted by convergent extension 
movement which shape the developing embryo during gastrulation [Shook et al., 2018]. This connection has only recently been made by studies in Xenopus. Chien et al. [2018] have explanted presumptive skin or LRO tissues and applied artificial strain to explants, i.e., they have sucked explants into glass capillaries to mimic gastrulation strain forces, which would not have been possible in any other vertebrate model [Chien et al., 2015; Blum and Ott, 2018a]. Their work beautifully and convincingly showed that strain is required for generation of the PCP vector, for cilia lengthening, and motility. These forces in all likelihood impact on ciliopathies such as PCD as well. No candidate gene has yet been assessed in that way, but such an analysis is easily performed in Xenopus.

We are in the process of analyzing a set of target genes of foxj1, which the Gossler laboratory in Hannover (Germany) has identified in mouse as PCD candidate genes [Weidemann et al., 2016; Stauber et al., 2017]. Interestingly, knock-down of some of these genes show clear ciliarelated phenotypes in Xenopus even though genetically null mice are completely normal [unpubl. data from Achim Gossler, T.O. and M.B.]. These findings implicate genetic compensation by family members in null mutations, a phenomenon first described in zebrafish [Rossi et al., 2015] and commonly acknowledged in mice. This finding also underscores that for assessing human genetic disease mechanisms, more than one model organism should be used for the cautious interpretation of experimental data.

\section{Conclusion and Perspectives}

The frog Xenopus has contributed in a major way to our understanding of basic embryological and cell biological principles of development, from the pluripotency of the somatic cell nucleus to the elucidation of Spemann organizer and major signaling pathways. The experimental and molecular repertoire available to manipulate developmental interactions and developmentally relevant genes in a precise and controlled manner admits these species as full-fledged members of the small group of predictive animal models to study human disease mechanisms. We foresee that the frog will have a critically important role in biomedical research and encourage the clinical and genetic communities alike to utilize this model alongside the more common mouse and zebrafish. The challenges for fast and reliable assessment of candidate genes requires using all models in parallel and to carefully compare results. The frog community is ready to meet the demand and willing to collaborate and to contribute. The profit will be mutual for science and patients - this chance is not to be missed.

\section{Disclosure Statement}

We confirm that there are no conflicts of interest.

\section{References}

Amin, N.M., T.M. Greco, L.M. Kuchenbrod, M.M. Rigney, M.-I. Chung, J.B. Wallingford, I.M. Cristea, F.L. Conlon (2014) Proteomic profiling of cardiac tissue by isolation of nuclei tagged in specific cell types (INTACT). Development 141: 962-973.

Asashima, M., Y. Ito, T. Chan, T. Michiue, M. Nakanishi, K. Suzuki, K. Hitachi, K. Okabayashi, A. Kondow, T. Ariizumi (2009) In vitro organogenesis from undifferentiated cells in Xenopus. Dev Dyn 238: 1309-1320.

Aslan, Y., E. Tadjuidje, A.M. Zorn, S.-W. Cha (2017) High-efficiency non-mosaic CRISPRmediated knock-in and indel mutation in F0 Xenopus. Development 144: 2852-2858.

Bier, E., E.M. De Robertis (2015) Embryo development. BMP gradients: a paradigm for morphogen-mediated developmental patterning. Science 348: aaa5838.

Blitz, I.L. (2018) Primordial germ cell transplantation for CRISPR/Cas9-based leapfrogging in Xenopus. J Vis Exp 132: e56035.
Blum, M., P. Andre, K. Muders, A. Schweickert, A. Fischer, E. Bitzer, S. Bogusch, T. Beyer, H.W.M. van Straaten, C. Viebahn (2007) Ciliation and gene expression distinguish between node and posterior notochord in the mammalian embryo. Differentiation 75: 133146.

Blum, M., T. Beyer, T. Weber, P. Vick, P. Andre, E. Bitzer, A. Schweickert (2009) Xenopus, an ideal model system to study vertebrate leftright asymmetry. Dev Dyn 238: 1215-1225.

Blum, M., E.M. De Robertis, J.B. Wallingford, C. Niehrs (2015) Morpholinos: antisense and sensibility. Dev Cell 35: 145-149.

Blum, M., K. Feistel, T. Thumberger, A. Schweickert (2014a) The evolution and conservation of left-right patterning mechanisms. Development 141: 1603-1613.

Blum, M., T. Ott (2018a) The power of strain: organizing left-right cilia. Dev Cell 45: 277-279.

Blum, M., T. Ott (2018b) Animal left-right asymmetry. Curr Biol 28: R301-R304.
Blum, M., A. Schweickert, P. Vick, C.V.E. Wright, M.V. Danilchik (2014b) Symmetry breakage in the vertebrate embryo: when does it happen and how does it work? Dev Biol 393: 109123.

Bonnard, C., A.C. Strobl, M. Shboul, H. Lee, B. Merriman, S.F. Nelson, O.H. Ababneh, E. Uz, T. Güran, H., Kayseriliet al. (2012) Mutations in IRX5 impair craniofacial development and germ cell migration via SDF1. Nat Genet 44: 709-713.

Boskovski, M.T., S. Yuan, N.B. Pedersen, C.K. Goth, S. Makova, H. Clausen, M. Brueckner, M.K. Khokha (2013) The heterotaxy gene GALNT11 glycosylates Notch to orchestrate cilia type and laterality. Nature 504: 456-459.

Brooks, E.R., J.B. Wallingford (2014) Multiciliated cells. Curr Biol 24: R973-R982.

Brooks, E.R., J.B. Wallingford (2015) In vivo investigation of cilia structure and function using Xenopus. Methods Cell Biol 127: 131-159.

Brown, J.M., G.B. Witman (2014) Cilia and diseases. Bioscience 64: 1126-1137.
Xenopus: An Undervalued Model

Organism for Human Genetic Disease
Cells Tissues Organs 2018;205:303-313 DOI: $10.1159 / 000490898$ 
Bustamante-Marin, X.M., L.E. Ostrowski (2017) Cilia and mucociliary clearance. Cold Spring Harb Perspect Biol 9: a028241.

Chien, Y.-H., R. Keller, C. Kintner, D.R. Shook (2015) Mechanical strain determines the axis of planar polarity in ciliated epithelia. Curr Biol 25: 2774-2784.

Chien, Y.H., S. Srinivasan, R. Keller, C. Kintner (2018) Mechanical strain determines cilia length, motility and planar position in the left-right organizer. Dev Cell 45: 316-330.

Cruciat, C.-M., C. Niehrs (2013) Secreted and transmembrane Wnt inhibitors and activators. Cold Spring Harb Perspect Biol 5: a015081.

De Robertis, E.M., J. Larraín, M. Oelgeschläger, O. Wessely (2000) The establishment of Spemann's organizer and patterning of the vertebrate embryo. Nat Rev Genet 1: 171-181.

del Viso, F., F. Huang, J. Myers, M. Chalfant, Y. Zhang, N. Reza, J. Bewersdorf, C.P. Lusk, M.K. Khokha (2016) Congenital heart disease genetics uncovers context-dependent organization and function of nucleoporins at cilia. Dev Cell 38: 478-492.

Dubaissi, E., N. Papalopulu (2011) Embryonic frog epidermis: a model for the study of cellcell interactions in the development of mucociliary disease. Dis Model Mech 4: 179-192.

Dubaissi, E., K. Rousseau, R. Lea, X. Soto, S. Nardeosingh, A. Schweickert, E. Amaya, D.J. Thornton, N. Papalopulu (2014) A secretory cell type develops alongside multiciliated cells, ionocytes and goblet cells, and provides a protective, anti-infective function in the frog embryonic mucociliary epidermis. Development 141: 1514-1525.

Eisen, J.S., J.C. Smith (2008) Controlling morpholino experiments: don't stop making antisense. Development 135: 1735-1743.

Endicott, S.J., B. Basu, M. Khokha, M. Brueckner (2015) The NIMA-like kinase Nek2 is a key switch balancing cilia biogenesis and resorption in the development of left-right asymmetry. Development 142: 4068-4079.

Fakhro, K.A., M. Choi, S.M. Ware, J.W. Belmont, J.A. Towbin, R.P. Lifton, M.K. Khokha, M. Brueckner (2011) Rare copy number variations in congenital heart disease patients identify unique genes in left-right patterning. Proc Natl Acad Sci USA 108: 2915-2920.

Fliegauf, M., T. Benzing, H. Omran (2007) When cilia go bad: cilia defects and ciliopathies. Nat Rev Mol Cell Biol 8: 880-893.

Garcia de la Serrana, D., E.A. Mareco, I.A. Johnston (2014) Systematic variation in the pattern of gene paralog retention between the teleost superorders Ostariophysi and Acanthopterygii. Genome Biol Evol 6: 981-987.

Garfinkel, A.M., M.K. Khokha (2017) An interspecies heart-to-heart: Using Xenopus to uncover the genetic basis of congenital heart disease. Curr Pathobiol Rep 5: 187-196.

Gentsch, G.E., T. Spruce, R.S. Monteiro, N.D.L. Owens, S.R. Martin, J.C. Smith (2018) Innate immune response and off-target Mis-splicing are common morpholino-induced side effects in Xenopus. Dev Cell 44: 597-610.e10.
Gerdes, J.M., E.E. Davis, N. Katsanis (2009) The vertebrate primary cilium in development, homeostasis, and disease. Cell 137: 32-45.

Getwan, M., S.S. Lienkamp (2017) Toolbox in a tadpole: Xenopus for kidney research. Cell Tissue Res 369: 143-157.

Gordon, C.T., S. Xue, G. Yigit, H. Filali, K. Chen, N. Rosin, K.-I. Yoshiura, M. Oufadem, T.J. Beck, R. McGowanet al. (2017) De novo mutations in SMCHD1 cause bosma arhinia microphthalmia syndrome and abrogate nasal development. Nat Genet 49: 249-255.

Griffin, J.N., F. del Viso, A.R. Duncan, A. Robson, W. Hwang, S. Kulkarni, K.J. Liu, M.K. Khokha (2018) RAPGEF5 regulates nuclear translocation of $\beta$-catenin. Dev. Cell 44: 248-260.e4.

Gurdon, J.B., T.R. Elsdale, M. Fischberg (1958) Sexually mature individuals of Xenopus laevis from the transplantation of single somatic nuclei. Nature 182: 64-65.

Gurdon, J.B., N. Hopwood (2000) The introduction of Xenopus laevis into developmental biology: of empire, pregnancy testing and ribosomal genes. Int J Dev Biol 44: 43-50.

Hagenlocher, C., P. Walentek, C. Müller, T Thumberger, K. Feistel (2013) Ciliogenesis and cerebrospinal fluid flow in the developing Xenopus brain are regulated by foxj1. Cilia 2: 12.

Hamburger, V. (1988) The Heritage of Experimental Embryology - Hans Spemann and the Organizer. New York, Oxford University Press.

Harland, R.M., M.J. Gilchrist (2017) Editorial: The Xenopus laevis genome. Dev Biol 426: 139-142.

Heasman, J. (2002) Morpholino oligos: making sense of antisense? Dev Biol 243: 209-214.

Hellsten, U., R.M. Harland, M.J. Gilchrist, D. Hendrix, J. Jurka, V. Kapitonov, I. Ovcharenko, N.H. Putnam, S. Shu, L. Taheret al. (2010) The genome of the Western clawed frog Xenopus tropicalis. Science 328: 633-636.

Hoff, S., J. Halbritter, D. Epting, V. Frank, T.-M.T Nguyen, J. van Reeuwijk, C. Boehlke, C. Schell, T. Yasunaga, M. Helmstädter et al. (2013) ANKS6 is a central component of a nephronophthisis module linking NEK8 to INVS and NPHP3. Nat Genet 45: 951-956.

Hosoya, M., Y. Kunisada, A. Kurisaki, M. Asashima (2012) Induction of differentiation of undifferentiated cells into pancreatic beta cells in vertebrates. Int J Dev Biol 56: 313-323.

Inaba, K., K. Mizuno (2016) Sperm dysfunction and ciliopathy. Reprod Med Biol 15: 77-94.

Jacobson, M., S.A. Moody (1984) Quantitative lineage analysis of the frog's nervous system. I. Lineages of Rohon-Beard neurons and primary motoneurons. J Neurosci 4: 1361-1369.

Keller, R. (2002) Shaping the vertebrate body plan by polarized embryonic cell movements. Science 298: 1950-1954.

Keller, R., L. Davidson, A. Edlund, T. Elul, M. Ezin, D. Shook, P. Skoglund (2000) Mechanisms of convergence and extension by cell intercalation. Philos Trans R Soc Lond B Biol Sci 355: 897-922.
Knowles, M.R., M. Zariwala, M. Leigh (2016) Primary ciliary dyskinesia. Clin Chest Med 37: 449-461.

Kok, F.O., M. Shin, C.-W. Ni, A. Gupta, A.S. Grosse, A. van Impel, B.C. Kirchmaier, J. Peterson-Maduro, G. Kourkoulis, I. Male et al. (2015) Reverse genetic screening reveals poor correlation between morpholino-induced and mutant phenotypes in zebrafish. Dev Cell 32: 97-108.

Kurisaki, A., Y. Ito, Y. Onuma, A. Intoh, M. Asashima (2010) In vitro organogenesis using multipotent cells. Hum Cell 23: 1-14.

Lienkamp, S.S. (2016) Using Xenopus to study genetic kidney diseases. Semin Cell Dev Biol 51: 117-124.

Lienkamp, S., A. Ganner, C. Boehlke, T. Schmidt, S.J. Arnold, T. Schäfer, D. Romaker, J. Schuler, S. Hoff, C. Powelske et al. (2010) Inversin relays Frizzled-8 signals to promote proximal pronephros development. Proc Natl Acad Sci USA 107: 20388-20393.

Lienkamp, S.S., K. Liu, C.M. Karner, T.J. Carroll, O. Ronneberger, J.B. Wallingford, G Walz (2012) Vertebrate kidney tubules elongate using a planar cell polarity-dependent, rosettebased mechanism of convergent extension. Nat Genet 44: 1382-1387.

MacArthur, D.G., T.A. Manolio, D.P. Dimmock, H.L. Rehm, J. Shendure, G.R. Abecasis, D.R. Adams, R.B. Altman, S.E. Antonarakis, E.A. Ashleyet al. (2014) Guidelines for investigating causality of sequence variants in human disease. Nature 508: 469-476.

Marshall, W.F., S. Nonaka (2006) Cilia: tuning in to the cell's antenna. Curr Biol 16: R604R614.

Mitchison, H.M., E.M. Valente (2017) Motile and non-motile cilia in human pathology: from function to phenotypes. J Pathol 241: 294309.

Moody, S.A. (1987) Fates of the blastomeres of the 32-cell-stage Xenopus embryo. Dev Biol 122: 300-319.

Moody, S.A. (2000) Cell lineage analysis in Xenopus embryos. Methods Mol Biol 135: 331347.

Moreno-Mateos, M.A., J.P. Fernandez, R. Rouet, C.E. Vejnar, M.A. Lane, E. Mis, M.K. Khokha, J.A. Doudna, A.J. Giraldez (2017) CRISPRCpf1 mediates efficient homology-directed repair and temperature-controlled genome editing. Nat Commun 8: 2024

Moulton, J.D. (2017) Making a morpholino experiment work: controls, favoring specificity, improving efficacy, storage, and dose. Methods Mol Biol 1565: 17-29.

Naert, T., T. van Nieuwenhuysen, K. Vleminckx (2017) TALENs and CRISPR/Cas9 fuel genetically engineered clinically relevant Xenopus tropicalis tumor models. Genesis 55: e23005.

Naert, T., K. Vleminckx (2018) CRISPR/Cas9mediated knockout of Rb1 in Xenopus tropicalis. Methods Mol Biol 1726: 177-193. 
Nagasawa, K., Y. Tanizaki, T. Okui, A. Watarai, S. Ueda, T. Kato (2013) Significant modulation of the hepatic proteome induced by exposure to low temperature in Xenopus laevis. Biol Open 2: 1057-1069.

Peshkin, L., M. Wühr, E. Pearl, W. Haas, R.M. Freeman Jr, J.C. Gerhart, A.M. Klein, M. Horb, S.P. Gygi, M.W. Kirschner (2015) On the relationship of protein and mRNA dynamics in vertebrate embryonic development. Dev Cell 35: 383-394.

Philpott, A., P.R. Yew (2008) The Xenopus cell cycle: an overview. Mol Biotechnol 39: 9-19.

Reversade, B., N. Escande-Beillard, A. Dimopoulou, B. Fischer, S.C. Chng, Y. Li, M. Shboul, P.-Y. Tham, H. Kayserili, L. Al-Gazali et al. (2009) Mutations in PYCR1 cause cutis laxa with progeroid features. Nat Genet 41: 10161021.

Rossi, A., Z. Kontarakis, C. Gerri, H. Nolte, S Hölper, M. Krüger, D.Y.R. Stainier (2015) Genetic compensation induced by deleterious mutations but not gene knockdowns. Nature 524: 230-233.

Samocha, K.E., E.B. Robinson, S.J. Sanders, C. Stevens, A. Sabo, L.M. McGrath, J.A. Kosmicki, K. Rehnström, S. Mallick, A. Kirby et al. (2014) A framework for the interpretation of de novo mutation in human disease. Nat Genet 46: 944-950.

Session, A.M., Y. Uno, T. Kwon, J.A. Chapman, A. Toyoda, S. Takahashi, A. Fukui, A. Hikosaka, A. Suzuki, M. Kondo et al. (2016) Genome evolution in the allotetraploid frog $\mathrm{Xe}$ nopus laevis. Nature 538: 336-343.
Shinohara, K., H. Hamada (2017) Cilia in leftright symmetry breaking. Cold Spring Harb Perspect Biol 9: a028282.

Shook, D.R., E.M. Kasprowicz, L.A. Davidson, R. Keller (2018) Large, long range tensile forces drive convergence during Xenopus blastopore closure and body axis elongation. Elife 7: 115.

Stauber, M., M. Weidemann, O. Dittrich-Breiholz, K. Lobschat, L. Alten, M. Mai, A. Beckers, M. Kracht, A. Gossler (2017) Identification of FOXJ1 effectors during ciliogenesis in the foetal respiratory epithelium and embryonic left-right organiser of the mouse. Dev Biol 423: 170-188.

Szabó, A., M. Melchionda, G. Nastasi, M.L. Woods, S. Campo, R. Perris, R. Mayor (2016) In vivo confinement promotes collective migration of neural crest cells. J Cell Biol 213: 543-555.

Tandon, P., F. Conlon, J.D. Furlow, M.E. Horb (2017) Expanding the genetic toolkit in Xenopus: approaches and opportunities for human disease modeling. Dev Biol 426: 325-335.

Vick, P., A. Schweickert, T. Weber, M. Eberhardt, S. Mencl, D. Shcherbakov, T. Beyer, M. Blum (2009) Flow on the right side of the gastrocoel roof plate is dispensable for symmetry breakage in the frog Xenopus laevis. Dev Biol 331: 281-291.
Walentek, P., S. Bogusch, T. Thumberger, P. Vick, E. Dubaissi, T. Beyer, M. Blum, A. Schweickert (2014) A novel serotonin-secreting cell type regulates ciliary motility in the mucociliary epidermis of Xenopus tadpoles. Development 141: 1526-1533.

Walentek, P., I.K. Quigley (2017) What we can learn from a tadpole about ciliopathies and airway diseases: using systems biology in Xenopus to study cilia and mucociliary epithelia. Genesis 55: e23001.

Weidemann, M., K. Schuster-Gossler, M. Stauber, C. Wrede, J. Hegermann, T. Ott, K. Boldt, T. Beyer, K. Serth, E. Kremmer et al. (2016) CFAP157 is a murine downstream effector of FOXJ1 that is specifically required for flagellum morphogenesis and sperm motility. Development 143: 4736-4748.

Wühr, M., R.M. Freeman, M. Presler, M.E. Horb, L. Peshkin, S.P. Gygi, M.W. Kirschner (2014) Deep proteomics of the Xenopus laevis egg using an mRNA-derived reference database. Curr Biol 24: 1467-1475.

Wühr, M., T. Güttler, L. Peshkin, G.C. McAlister, M. Sonnett, K. Ishihara, A.C. Groen, M. Presler, B.K. Erickson, T.J. Mitchison et al. (2015) The nuclear proteome of a vertebrate. Curr Biol 25: 2663-2671.

Xiao, Q., Z. Chen, X. Jin, R. Mao, Z. Chen (2017) The many postures of noncanonical Wnt signaling in development and diseases. Biomed Pharmacother 93: 359-369.

Zariwala, M.A., M.R. Knowles, H. Omran (2007) Genetic defects in ciliary structure and function. Annu Rev Physiol 69: 423-450. 